DOI: 10.12957/demetra.2018.33525

\title{
Precariedad y alimentación: las redes sociales y de apoyo en los comedores sociales
}

\section{Precariousness and food: social and support networks in social canteens}

Ursula Verthein

1 Universidad de Barcelona, Facultad de Geografía e Historia, Departamento de Antropología Social. Barcelona, España.

Este artículo se deriva de la tesis doctoral en Alimentación y Nutrición presentada por la autora de este artículo en el Departamento de Antropología Social de la Facultad de Geografía e Historia de la Universidad de Barcelona.

Correspondencia / Correspondence Ursula Verthein

E-mail: ursulaverthein@gmail.com

\section{Resumen}

Este estudio se desarrolla en contexto de crisis económica que afecta a Europa, y consecuentemente, al Estado español, a partir del año 2008. Y analiza, a través de un enfoque etnográfico, las formas de reconfiguración de las redes sociales de apoyo de personas que utilizan servicios asistenciales para alimentación, como comedores sociales, en la ciudad de Barcelona (España). Analizamos especialmente qué funciones y responsabilidades asumen los comedores sociales cuando los antiguos lazos de referencia de ayuda (familiares y amigos) de las personas que utilizan estos centros como recurso se han debilitado como consecuencia del proceso de empobrecimiento que atraviesan. Interesa entender hasta qué punto las políticas públicas para la alimentación pueden y deben considerar la dimensión social (y afectiva) en sus planteamientos y acciones, ya que, en muchos casos, los centros asistenciales y las personas que trabajan allí pasan a ser para sus usuarios los lugares e individuos de referencia en este sentido. Hemos identificado que los comedores sociales, aunque por veces manifiesten su función de promover relaciones sociales entre las personas que los utilizan, no siempre valoran con profundidad la cuestión, lo que plantea la necesidad de discutir las problemáticas que surgen a partir de la constatación de este dato.

Palabras clave: Asistencia alimentaria. Pobreza. Relaciones familiares. España. 


\section{Abstract}

The current study was carried out in the economic crisis context that has been affecting Europe and, consequently, Spain since 2008. Based on the ethnographic approach, it analyzes the reconfiguration of social networks focused on supporting people who seek welfare services such as social canteens to get food in Barcelona City (Spain). We mainly analyzed the functions and responsibilities taken by social canteens after the old aid-reference bonds (family and friends) of people who use these centers as food resource weakened due to the impoverishment process they went through. The aim is to understand to what extent public policies focused on Right to Food can and should take into consideration the social (and affective) dimension in their approaches and actions, since, in many cases, welfare service centers, and people who work in them, become reference for that matter. Based on our analysis, although sometimes social canteens promote social relations among people using their services, they do not always value this matter in a comprehensive way, fact that opens room for issues that need to be discussed.

Keywords: Food Assistance. Poverty. Family Relationships. Spain.

\section{Introducción}

Este estudio se desarrolla en un contexto de crisis económica que afecta a Europa, y consecuentemente al Estado español, a partir del año 2008. Según los datos del Instituto Nacional de Estadística español, ${ }^{1}$ el riesgo de pobreza y exclusión social de la población española era del 24,7\% en 2009; del 26,1\% en 2010 y del 29,2\% en 2014. El agravamiento de la crisis también se fue profundizando en Cataluña. Según el Institut d'Estadística de Catalunya ${ }^{1}$ (Idescat), el riesgo de pobreza de las personas entre 16 y 64 años aumentó del 17\% en 2009, al 18,4\% en 2011 y al 20,6\% en 2014.

El proceso de precarización de la vida que surge como consecuencia de la situación socioeconómica de crisis e desigualdad social en las sociedades capitalistas a nivel local y global ha transformado las prácticas de consumo y los modos de vida de tal forma que, en los últimos años, se han identificado transformaciones diversas en relación a cómo consumimos, nos relacionamos, nos organizamos políticamente, construimos nuestras relaciones afectivas, entre otras transformaciones a nivel individual y colectivo, tanto en la esfera pública como en la esfera privada.-8

Por este motivo, este artículo analiza las formas de reconfiguración de las redes sociales y de apoyo de personas que sufren procesos de precarización social y económica en la ciudad de 
Barcelona (España). Específicamente, observamos las transformaciones que han ocurrido en las relaciones de personas que utilizan el servicio asistencial de comedores sociales con sus antiguas redes de ayuda (familiares y amigos). Las mismas manifestaron que, en función de su actual situación de dependencia económica, se habían debilitado o extinguido los lazos afectivos con familiares y amigos que habían sido siempre considerados como sus referencias de ayuda y afecto.

Interpretamos, pues, cómo se han reconfigurado estas redes sociales, analizando especialmente qué papel asumen los servicios asistenciales para la alimentación (específicamente los comedores sociales) en estas circunstancias. Interesa entender hasta qué punto las políticas públicas pueden y deben considerar la dimensión social (y afectiva) en sus planteamientos y acciones, ya que, en muchos casos, los centros asistenciales y las personas que trabajan allí pasan a ser los lugares e individuos de referencia en este sentido, según manifestaron en nuestro trabajo de campo las personas que los utilizan.

Durante nuestro trabajo de campo se puso de manifiesto que la totalidad de las personas usuarias $^{\text {a }}$ de los comedores sociales entrevistadas mencionaron haber sufrido la reconfiguración de sus redes sociales y de apoyo como consecuencia del inicio de los procesos de precarización de vida que afrontaban. Identificaron que el empobrecimiento y sus consecuencias directas e indirectas (afectaciones anímicas, incapacidad de pagar por momentos de ocio y situaciones de convivialidad social, deseo de autoexclusión de situaciones de sociabilidad, etc.) habían afectado la manera como se relacionaban con las personas con las que habían mantenido vínculos afectivos y de sociabilidad hasta entonces. Principalmente, destacaron: a) el alejamiento en relación a los antiguos lazos familiares y de amistad; b) la configuración de nuevos vínculos con personas que se encontraban en la misma situación económica de vulnerabilidad; c) que las redes de auxilio y apoyo habituales comúnmente habían dejado de ser la familia y los amigos y pasaron a ser las instituciones sociales de ayuda que utilizaban como recurso (servicios sociales, comedores sociales, albergues, etc.).

Un dato importante se refiere a que, tanto la institución (a través del discurso de los trabajadores de los centros) como los propios usuarios, concuerdan en que el debilitamiento de los antiguos vínculos afectivos y de ayuda agravan la situación de dependencia de recursos asistenciales por parte de las personas que sufren procesos de precarización porque las mismas ven reducidas sus posibilidades de respuesta cuando solicitan auxilio a familiares y amigos. El desgaste o aflojamiento de las relaciones afectivas que suele ser resultado de los procesos de empobrecimiento agrava la situación de dependencia y vulnerabilidad que afrontan las personas, según estas mismas manifestaron.

a El término "usuaria/o" es utilizado por la institución para referirse a las personas que utilizan el servicio de los comedores sociales. En este sentido, reproducimos la palabra. 
Diferentes estudios (Cernadas ${ }^{9}$; Max-Neef ${ }^{10}$; Pomar y Tendero ${ }^{11}$ ) también han relacionado la pobreza como causa del debilitamiento de las relaciones sociales de los individuos. De acuerdo con estos análisis, las reducidas, poco estables o nulas redes de ayuda derivadas del empobrecimiento, aumentan el riesgo de vulnerabilidad y desprotección de las personas, porque éstas ven disminuidas las respuestas de auxilio o acogimiento en caso de necesidad. Sobre este asunto, en su definición sobre el concepto de pobreza, Max Neef sostiene que no es posible hablar de ésta sino en plural ("pobrezas"). El autor entiende que cualquier necesidad humana fundamental, que no sea satisfecha adecuadamente, revela una modalidad de carencia o pobreza. Por ejemplo:

la pobreza de subsistencia (debido a alimentación y abrigo insuficientes); de protección (debido a sistemas de salud ineficientes, a la violencia, la carrera armamentista, etc.); de afecto (debido al autoritarismo, la opresión, las relaciones de explotación con el medio ambiente natural, etc.); de entendimiento (debido a la deficiente calidad de la educación); de participación (debido a la marginación y discriminación de mujeres, niños y minorías); de identidad (debido a la imposición de valores extraños a culturas locales y regionales, emigración forzada, exilio político, etc.) y así sucesivamente. (Max-Neef ${ }^{10}$ p.43).

Por ello, Max-Neef entiende como una forma de pobreza el aislamiento social derivado de los procesos de precarización. La necesidad de protección, afecto o entendimiento, manifestados por los usuarios de los comedores sociales como aspectos que no son suficientemente atendidos en sus vidas con posterioridad a la entrada en la precariedad, según su análisis, serían necesidades humanas (o pobrezas) comúnmente no consideradas como tal en nuestra sociedad, que fundamentalmente hace referencia a la pobreza económica. El autor denuncia, así, la necesidad de poner de manifiesto las carencias citadas como forma de hacerlas visibles. Afirma, además, que "cada pobreza genera patologías". Con esto quiere decir que, en función de los límites de intensidad y duración de la situación de precariedad en la que vive un individuo, las consecuencias en sus vidas se expresan como situaciones patológicas. Entre éstas, el miedo, la violencia y la marginación se destacan como "patologías sociales colectivas", que los diversos sistemas socio-políticos son responsables de provocar como resultado del bloqueo sistemático que ejercen sobre las necesidades humanas ya citadas.

Agurto, Arteaga y Pérez, ${ }^{12}$ a partir de un estudio sobre las subjetividades en familias urbanas económicamente vulnerables, sostienen que los grupos familiares que atraviesan procesos de precarización "despliegan un conjunto de acciones para enfrentar los quiebres socioeconómicos, pero además de orientarse a superar dichos eventos, se desarrollan para resolver otros tipos de crisis que emergen paralelamente a estas situaciones". (p. 109). Con ello, las autoras se refieren a un conjunto de afectaciones anímicas individuales y del grupo como red, en función de los sufrimientos generados por la percepción del riesgo. Con respecto a la comunidad, influye "en su capacidad de reacción ante los cambios del capital social”, es decir, en la confianza, y en las redes de reciprocidad: 
A partir del evento de quiebre de ingresos no sólo se genera una crisis ligada al aspecto económico, que es la más visible y fácil de identificar para las familias, sino que también se ponen en riesgo otros aspectos que se intentan proteger, mantener y/o restituir. A la primera crisis se le denominó crisis socioeconómica, mientras que a la segunda, crisis psicosocial. ${ }^{12}$ (p. 109)

Las autoras analizan, además, aquellos sentimientos y emociones que se generan producto de la situación de crisis y sus efectos. En su análisis identificaron como efectos asociados al evento crítico, la "angustia, tristeza y desesperación" individuales, y en consecuencia, mencionaron que "la crisis desarma afectivamente a la familia, lo que muchas veces genera roces, discusiones y quiebres en la dinámica familiar." ${ }^{12}$ (p. 109)

\section{Consideraciones metodológicas}

El estudio interpretó, a través de un enfoque cualitativo, el funcionamiento general de comedores sociales en la zona metropolitana de la ciudad de Barcelona (España). Con especial atención, describimos y analizamos la percepción de los usuarios sobre aspectos diversos del servicio que frecuentaban (por ejemplo, en relación a la calidad de las preparaciones servidas, a la relación con la institución, a la relación entre usuarios y a situaciones de convivialidad).

El estudio se desarrolló a través de una aproximación etnográfica. Así, el trabajo de campo, dividido en tres diferentes etapas entre los años de 2012 y 2015 se realizó en tres comedores sociales. El trabajo de campo consistió en observación directa y realización de entrevistas informales y formales (abiertas y en profundidad) con gestores, trabajadores y usuarios de los comedores sociales. Para la realización de entrevistas, elaboramos previamente guiones orientativos a partir de los interrogantes establecidos según nuestros objetivos de investigación.

Todas las personas entrevistadas dieron su consentimiento para la utilización de la información que nos transmitieron en el estudio. Se informó a cada una de éstas personas que no utilizaríamos sus nombres reales para preservarles de cualquier inconveniente relacionado con la publicación de la información que nos concedieron.

\section{Resultados}

Durante nuestro trabajo de campo, la dependencia (económica, de cuidados específicos en función de alguna enfermedad, etc.) fue señalada por las personas entrevistadas como el principal motivo responsable del crecimiento de la distancia entre los beneficiarios de los centros asistenciales y aquellas personas que siempre habían considerado como vínculos de auxilio frente a las adversidades (familiares y amigos). En estos casos, el proceso de alejamiento puede realizarse desde dos diferentes vías: desde la persona en situación de vulnerabilidad hacia sus lazos de 
afinidad anteriores, o desde éstos hacia las personas dependientes. Por una parte, la "vergüenza de depender" genera una distancia voluntaria de los individuos en situación de vulnerabilidad, en relación a sus redes.

A este respecto, entre los motivos mencionados, se destacan la afectación de la autoestima (y sus consecuencias en relación a la disminución del deseo de frecuentar situaciones sociales) y el propósito voluntario de desvinculación de personas "del pasado", con el fin de poder ocultar la actual situación económica de precariedad. Por otra parte, las personas en situación de dependencia se refieren a la falta de respuestas y al distanciamiento en general en relación a sus antiguos lazos y lo que atribuyen a una forma de que estas personas "eviten problemas en sus vidas". La "decepción" motivada por la negación de ayuda de familiares y amigos era un sentimiento repetido en las entrevistas y nos revelaba que la pobreza interviene en las relaciones afectivas debilitando los vínculos considerados "fuertes" o "sólidos" cuando ésta no existía.

Francisco identificaba el alejamiento de su familia hacia él, en función de su empobrecimiento, señalando que:

La familia se ha portado muy mal conmigo. Tengo una amistad, un matrimonio que conocí en el barrio, que son los que me vienen a ver en el hospital. Los cuatro meses que estuve solo han venido ellos. Y este amigo llamó a mis primos: “oiga, que su primo está en el hospital”. No tuvieron tiempo de venir. En cuatro meses no tuvieron un día. Él estando jubilado, el hijo también podría porque era época de verano, época de vacaciones. Y esto ocurre porque ya no soy el primo bueno, el que les llevaba los regalos. Hay mucho interés en la familia. [...] Hay mucho interés.

Josep cuenta que, desde hace cuatro años, no recibe noticias de su única hija y explica esta situación como consecuencia de que se vio imposibilitado de seguir manteniéndola económicamente después de la pérdida de su trabajo. Entiende que su empobrecimiento generó la actual falta de interés por parte de ella para relacionarse con él. En su discurso, señala directamente la condición de ofrecerle dinero para mantener el vínculo con su hija.

No sé nada de ella desde hace cuatro años. Ella no quiere saber nada de mí. Yo llevaba muy bien el tema laboral, me ganaba muy bien la vida y mi ex mujer me incitó a que ella me pidiera dinero: -Papá no tengo dinero, no tengo dinero. Cuando me quedé sin trabajo, le dije que no podía darle más dinero. Y su respuesta fue desaparecer. [...] No sé si está casada, si tiene trabajo, si tiene hijos. Le deseo lo mejor. Es un mal que traigo encima. Supongo que si algún día cambio y me caen 5 millones de euros, a lo mejor me dice: - Papá, estás vivo? Esta es mi historia.

En este sentido, Matulic ${ }^{4}$ (p.9.) sostiene que "los factores que inciden en el proceso de desvinculación social de las personas parten de una combinación y asociación de elementos individuales y estructurales que necesitan, a la vez, de una mirada micro y macro social del 
fenómeno". Así, la autora corrobora el discurso expresado por los usuarios de los comedores sociales, cuando afirman que las causas del aislamiento que sufren es multicausal. En sus respuestas, algunas personas mencionaban exclusivamente sus situaciones personales como las responsables de dicho proceso. Cuando esto ocurría, solían responsabilizarse a sí mismas de la soledad y del sentimiento de desprotección que manifestaban sentir. Por ejemplo, un usuario de unos de los comedores que realizamos trabajo de campo se refería a que, actualmente, había perdido sus redes de apoyo y que, por esa razón, ya no podía recurrir a ninguna persona de su familia. Cuando se le preguntó por la causa de dicha situación, responsabilizó al "proceso de encierro" que sufrió en los últimos años como consecuencia del empobrecimiento: "Ahora no tengo a nadie porque yo prácticamente me he encerrado en mí mismo y ya me cuesta tener apoyo".

Otras veces, algunas personas identificaban cuestiones sociales externas a sus vidas personales como las responsables de un proceso de individualización social que, en general, se refleja en su autopercepción como personas desprotegidas, "solas" y "vulnerables". En este sentido, José mencionaba las consecuencias del proceso de individualización que siente en su vida particular en la actualidad, en comparación con el pasado, como un fenómeno social. Por ejemplo, citaba que las situaciones en las que celebraba fechas festivas con su familia se habían extinguido y consideraba que la causa se debía a los cambios sociales "naturales" que existen entre las diferentes generaciones:

No es que lo diga yo. Yo lo veo. Antiguamente nos veíamos la familia, quedábamos en fin de año, un fiestorro en casa, a bailar. Lo clásico, y te reías. Yo creo que va pasando el tiempo y la vida va cambiando. Las generaciones son de otra manera, otro rollo.

Ángela identifica la afirmación expresada por Matulic sobre la relación entre aspectos particulares y generales (micro y macro sociales) como causa del aislamiento general de los individuos en nuestra sociedad. Inicialmente, reconoce que, en el presente, hay un aumento del individualismo en relación al pasado. Para justificar su afirmación, menciona una serie de ejemplos: a través de las diferencias en cómo nos reunimos para charlar cotidianamente, las formas que utilizábamos y las que utilizamos para felicitarnos, en cómo comprábamos los alimentos y cómo lo hacemos ahora, entre otras. Posteriormente, señala cómo dicho proceso de individualización influye en nuestra autopercepción e identidad individual, creyendo que actualmente somos "más cerrados" y tenemos "más necesidad de protegernos" de los demás. Finalmente, entiende que el proceso general de aislamiento social es responsable de influir en la manera en cómo nos queremos a nosotros mismos:

Sí que había más unión que ahora. Yo me acuerdo que las madres se sentaban en la calle haciendo medias, ganchillo y hablaban que si de la una que si de la otra, y los críos estábamos en la calle jugando. Esto ahora ya no existe. Nos felicitábamos por carta postal y esto ahora con los móviles e internet, nada. Íbamos a los colmados, a las tiendas pequeñas y ya sabían lo que querías y lo que no querías: 
"oye, guárdame tal cosa..." Y ahora vas a una gran superficie y la persona que te está atendiendo parece que tenga dolor de barriga, llevan una cara que dices... Se te quita las ganas incluso de decirle buenos días. O sea, nos estamos encerrando, estamos más individuales. Entonces te lleva a convertirte también de una manera, como para protegerte. Porque como seas demasiado abierto o simpático ya te toman el pelo. A mí me han echado de trabajos por defender a los compañeros. O sea, es todo un proceso en general, en todas partes, la familia, el trabajo, y creo que ya hemos llegado a no querernos ni a nosotros mismos. Si nosotros mismos nos quisiéramos, nos comportaríamos de otra manera, para estar con gente, compartir, ser solidarios. Pero llega un momento en que uno mismo ya no se quiere ya, todo le da igual, se encierra.

Vale recordar que algunos autores (Bourdieu, ${ }^{13}$ Bauman²) han identificado el fenómeno creciente de reconfiguración de las redes sociales en nuestra sociedad. Las consecuencias directas del aislamiento social en los diferentes aspectos de la vida, son interpretadas por Castel ${ }^{14}$ al analizar lo que entiende por "desafiliación". Según este autor, este proceso ocurre "cuando el conjunto de las relaciones de proximidad que mantiene un individuo sobre la base de su inscripción territorial, que es también su inscripción familiar y social, tiene una falla". Siguiendo su lectura, la "desafiliación" es responsable de "impedir la existencia” y comprometer los mecanismos generales de "protección" de las personas.

Por lo que se refiere a las relaciones de parentesco como fuente de apoyo en situaciones de vulnerabilidad, las personas entrevistadas identificaron que los progenitores eran sus referencias de ayuda y acogimiento. Con el fallecimiento de los padres y suegros, las redes familiares se debilitaron y las respuestas frente a las solicitudes de ayuda se redujeron visiblemente. Los hermanos y los hijos habitualmente son mencionados como entes que "fallan" o "decepcionan" ya que no suelen corresponder a las expectativas de acogimiento cuando las personas que sufren procesos de precarización lo solicitan. Sobre este aspecto se ha evidenciado también, una prevalencia de "abandono" de los hijos en los casos relatados por las personas mayores. José identifica al padre como la persona que le acogió tras el desahucio de su vivienda. La madre y la suegra también fueron personas que le demostraron afecto, pero con el fallecimiento de estas personas, José expresa sentir la pérdida de sus principales referentes de atención y cuidado.

Me casé, tuve mi casa. Bueno, hubo un desahucio y después me recogió mi padre. Se murió mi padre, estoy en una habitación. [...] Mi suegra era como si fuera mi segunda madre. A veces se enfadaban las dos. Mi madre y mi suegra. Porque si yo me iba a comer a casa de mi suegra, estaban las dos como celosillas. Mi suegra me cuidaba mucho. Yo me iba a la cocina, ella me ponía mi cerveza y mis olivas con ajo. Y me hacía unos cocidos... Fíjate tú de lo que me he acordado ahora, una cosa muy buena. Cuando hacía cocido me llamaba: "vente que he hecho cocido". Me hacía unos cocidos para chuparse los dedos. Qué cocido más rico. 
Lourdes, una mujer de 81 años, relataba que, pese a que dedicó parte de su vida a criar a sus hijos y nietos, actualmente éstos no la visitan. Cuenta que, en situaciones en las que por enfermedad necesitó ayuda, recibió apoyo de una vecina, ya que sus parientes no acudieron. Atribuye la ausencia de sus familiares a una "falta de interés", ya que todos residen a pocos quilómetros de su residencia. En su caso, considera que la vejez, además de la situación de vulnerabilidad económica, han influido en el aislamiento y alejamiento de las personas que antes eran consideradas como vínculos cercanos.

Yo he tenido la desgracia de tener un cáncer de mama, estereotomía total, una prótesis de rodilla, ahora tengo este problema en la espalda, me rompí la pelvis, varias vértebras, y ahora ando muy mal. Todos los papeles que me he arreglado para tener una persona que me ayude a mí en mi casa me los he arreglado yo. Tengo problemas con mis hijos. Los hijos crecen, viven su vida, Aunque ahora ven como estás que ya te quedan pocos telediarios. Tengo una hija que [...] esta hija es que me mata. Me duele horrores. A ella no recurriría para nada. Cuando me rompí la pelvis porque me caí de una escalera y estuve en cama un mes y medio, no vino nadie a mi casa. Me cuidó una señora vecina y allí me enfrié mucho porque es que no vinieron, nadie...

En función de la reducción o disolución de las antiguas redes sociales, como pueden ser la familia y los amigos, las personas entrevistadas manifestaron crear nuevos vínculos como recurso de autoprotección y manutención de la sociabilidad. Habitualmente han manifestado que sus nuevas redes de apoyo se habían reconfigurado tras la conciencia de que sus antiguos lazos se habían distanciado de ellas. Tal reconfiguración se realiza, según mencionaron, a través de la aproximación de: a) personas que afrontan situaciones semejantes de precarización social y económica; b) las instituciones de asistencia social y sus trabajadores, que pasan a ser las referencias de apoyo y ayuda frente a situaciones diversas (cuestiones económicas, de carácter personal, problemáticas en lo que se refiere a su capacidad relacional, etc.).

Sobre la aproximación de personas del nuevo círculo social, Josep decía:

He conocido aquí [en el comedor social] una mujer. Hace dos meses y 12 días que somos pareja. Además, con varios amigos de aquí intentaremos encontrar un piso para cuatro o cinco personas. Un poquito de todos hace un muchito. Y es un paso más para un poco más de libertad.

De todos modos, algunas referencias a los vínculos que se establecen en los comedores sociales suelen indicar la superficialidad de las nuevas relaciones. María, por ejemplo, contaba que "una cosa es empatizar con estas personas, otra cosa es contarles mi vida, eso no. Nunca cuentes tus secretos porque te tienen cogida por los huevos. Tus defectos, paranoias, guárdalas".

Sobre la importancia de las instituciones como centros referenciales de ayuda a las personas, Castel $^{14}$ sostiene que el fortalecimiento de la estructura social que les concede el papel de apoyo 
y auxilio ("social-asistencial") puede caracterizarse formalmente por oposición a los modos de organización colectiva que evitan la necesidad de este tipo de recursos. Así, en las sociedades capitalistas urbanizadas, en las que predomina el debilitamiento de las acciones grupales y de formación espontánea y autónoma de redes de ayuda entre las personas, la asistencia se formaliza, se especializa y se tecnifica. Para el autor, en las ocasiones en que se reducen los vínculos colectivos no institucionalizados ("la sociabilidad primaria"), la asistencia a las personas es objeto de "prácticas especializadas"b, siendo el hospital, el orfanato, la distribución organizada de limosnas, instituciones "sociales" que colaboran con el "tratamiento particular (especial y especializado) de problemas que en sociedades menos diferenciadas eran asumidos sin mediación de la comunidad".

Según Castel, las características de la asistencia especializada son formales, porque corresponden a un proyecto de auxilio elaborado desde la institución. Por lo tanto, el objetivo de la asistencia institucional está en disminuir de manera organizada los efectos de la reducida o inexistente "sociabilidad primaria", desde un proyecto previo de actuación. Durante las entrevistas, algunos usuarios ratificaron la perspectiva defendida por Castel sobre la especialización y la tecnificación de la ayuda asistencial. Afirmaron que, ante la necesidad de solicitar auxilio, recurrían a los centros asistenciales que frecuentaban, desconsiderando la posibilidad de pedir ayuda a personas conocidas. En este caso concreto, los trabajadores de los centros asistenciales utilizados como recursos, fueron citados especialmente como las personas a quienes los entrevistados recurrían en diferentes situaciones de necesidad. En una entrevista, un usuario de unos de los comedores señaló que cuando tiene algún problema llama a "su" asistente social, quien le conoce por su nombre de pila, lo que indica un grado de proximidad. Mencionó que, al ser solicitada, esta respondía rápidamente a su demanda, ya fuese una cuestión personal o una necesidad material. Víctor, también usuario del servicio de comedor social, manifestaba que si se encontrase en cualquier situación de necesidad:

Recurriría al comedor, o llamaría a la asistente social. O sea, que es una buena asistente social, se llama Carmen. Porque ya me cuesta abrirme a todo el mundo. Aunque sean buenas personas, pero bueno, tener que contar mi vida ya me cuesta mucho. Cuando necesito alguna ayuda la llamo [a la asistente social] por teléfono, se pone enseguida y tal. Y le digo: He tenido este pequeño problema y quiero que me aconsejes. Y si ve que no lo puedo resolver, me dice: si te dicen que no pueden resolver me vienes a ver que yo te atiendo. Siempre responde, siempre está... Bien.

Cernadas ${ }^{9}$ llama la atención respecto a las problemáticas derivadas de las funciones y de las condiciones a las que están expuestas los trabajadores de los centros asistenciales. El autor entiende que elementos como "la presión, la falta de instalaciones adecuadas a veces, el código ético particular de cada profesional y la mayor o menor sensibilidad en relación con el colectivo",

b Cursiva del autor. 
pueden ser responsables de actuaciones que no garantizan el acceso y la permanencia adecuada y en condiciones de igualdad, de la totalidad de los usuarios. Sobre esto, Cernadas destaca que en su trabajo identificó que el aspecto externo del beneficiario condiciona el tipo de asistencia que los profesionales le dirigen:

Tanto los trabajadores del servicio de ambulancias, los vigilantes de los centros sanitarios, los médicos, enfermeras, etc.; de forma amplia se declaran influidos por el aspecto externo del usuario, de tal manera que si lleva ropa sucia o rota, si está sucio, si hace mal olor, si ha bebido y hace olor a alcohol, si no se expresa con una mínima claridad - por citar alguno de los aspectos más importantes -; muestran un cierto rechazo y este usuario puede acabar sin ninguna atención [...]. (Cernadas ${ }^{9}$, p. 16)

Resalta, además, que las dificultades se agravan porque, habitualmente, las personas que utilizan los recursos asistenciales suelen sentirse vulnerables y rechazadas tanto por las instituciones como por la sociedad en general, y porque según su percepción, "la administración sanitaria y los profesionales sanitarios forman parte de aquello que ellos consideran que los margina y los mantiene en esta situación de abandono" (Cernadas ${ }^{9}$, p. 18).

Así, algunos usuarios que entrevistamos también identificaron que la efectividad en la creación o profundización de los lazos afectivos que se construyen en los centros asistenciales tendría que presuponer la reducción o eliminación del estigma relacionado con la pobreza y con la utilización de estos servicios. En este sentido, según las mismas personas, las políticas asistenciales deberían promover y asegurar su autonomía y poder de decisión sobre los diversos aspectos del funcionamiento general de los servicios asistenciales que utilizan. Señalan los usuarios la importancia de que pudiesen participar activamente de las diferentes etapas y actividades de planteamiento y acción de los servicios que utilizan y que las acciones asistenciales tendrían que trabajar en el sentido de garantizar su derecho de actuar como sujetos políticos de los recursos asistenciales que frecuentan. Según las mismas personas, estas acciones serían, pues, también responsables de reducir sus afectaciones anímicas ("depresiones", "incomodidad" "vergüenza de depender", "tristeza”) porque crearían otro tipo de implicación y relación de las personas con los centros sociales que frecuentan. Entienden, así, que estos modelos de participación activa y no jerárquica entre usuarios e institución colaboraría en el establecimiento y consolidación de los lazos afectivos que se intentan promover desde las políticas públicas asistenciales, lo que no siempre es alcanzado con efectividad.

\section{Conclusiones}

En un contexto de crisis como el que se encuentra el Estado Español y, consecuentemente Cataluña, a partir del año 2008, se identifica un proceso de precarización de la vida de la 
población en general que ha transformado prácticas de consumo, formas de convivio social, formas de organización política, entre otras transformaciones a nivel público y privado. En este sentido, estudiamos de qué manera los cambios en las redes sociales y afectivas de las personas que sufrieron procesos de empobrecimiento en este contexto afectaron la forma cómo solicitaban auxilio y apoyo. Percibimos, según se ha revelado en nuestro trabajo de campo, que las redes de ayuda sufrieron reconfiguraciones en función del proceso de crisis que afrontaban estas personas.

Las personas usuarias de comedores sociales entrevistadas en nuestro estudio manifestaron que, como consecuencia de su situación de dependencia económica, se habían debilitado o extinguido aquellos lazos afectivos considerados como fuertes y sólidos hasta su ingreso en la situación de precariedad. Así, los familiares y las relaciones de amistad que habían sido hasta entonces sus referencias de ayuda y afecto em muchos casos dejaban de serlo. En este sentido, éstas personas identificaron que el empobrecimiento y sus consecuencias directas e indirectas eran la causa fundamental de esta situación de reconfiguración de sus redes sociales. Entienden que elementos como las afectaciones anímicas, la reducción del poder de consumo y el deseo habitual de autoexclusión de situaciones de sociabilidad en este contexto, eran responsables de la transformación de la manera como se relacionaban con las personas con las que habían mantenido vínculos afectivos y de sociabilidad hasta entonces. Mencionaron, también, en muchos casos, haber creado nuevos vínculos con otras personas que se encontraban en la misma situación económica de vulnerabilidad y dependencia y que sus actuales redes de auxilio y apoyo pasaron a ser las instituciones sociales de ayuda que utilizaban como recurso asistencial (servicios sociales, comedores sociales, albergues, etc.).

Por lo tanto, este dato demuestra que las políticas públicas asistenciales, en general, y para la alimentación en particular, pueden y deben considerar la promoción y consolidación de las dimensiones sociales y afectivas de las personas que utilizan éstos servicios en sus planteamientos y acciones. Según hemos identificado, (Verthein ${ }^{15}$ ) los servicios asistenciales no siempre valoran suficientemente la importancia de que en estos se promuevan o profundicen relaciones sociales y afectivas, o aún y valorándolo no alcanzan resultados efectivos.

En este sentido, las personas usuarias de los servicios señalaron que la institución debería priorizar: la reducción o eliminación del estigma habitualmente relacionado con la pobreza y con la utilización de estos recursos; la autonomía y poder de decisión de los usuarios sobre los diversos aspectos del funcionamiento general de los servicios asistenciales que utilizan; el establecimiento de una relación dialógica y no jerárquica entre usuarios e institución. Entienden, así, que estos modelos de participación activa y no jerárquica entre usuarios e institución facilitarían la promoción, consolidación y profundización de relaciones sociales y afectivas que por veces se intentan promover desde las políticas públicas asistenciales (como en el caso de comedores sociales), pero que no siempre se consigue lograr. 


\section{Referências}

1. Institut d'Estadística de Catalunya. Taxa de risc de pobresa després de les transferències socials. Per sexe i grups d'edat. [Acceso en 06 de abril de 2018.]. Disponible en: https://www. idescat.cat/ $\mathrm{pub} / \mathrm{id}=\mathrm{aec} \& \mathrm{n}=416$

2. Bauman Z. Modernidad líquida. Buenos Aires: Fondo de Cultura Económica; 2003. Modernidad líquida. Buenos Aires: Fondo de cultura económica, 2003.

3. Baudrillard J. La sociedad de consumo: sus mitos, sus estructuras. Madrid: Siglo XXI; 2009.

4. Matulic M. Nuevos perfiles de personas sin hogar en la ciudad de Barcelona: un reto pendiente de los servicios sociales de proximidad. Revista de Trabajo y Acción Social. 2010; 48:9-30.

5. González-Turmo I, editora. Respuestas alimentarias a la crisis económica. Sevilla: ICAF Ebooks; 2013.

6. Gracia M. Comer en tiempos de crisis: nuevos contextos alimentarios y de salud en España. Salud Pública de México. 2014; 56(6):648-656.

7. Habermas J. Mudança estrutural da esfera pública. São Paulo: UNESP; 2014.

8. Sassen S. Una Sociología de la globalización. Análisis Político. sep.-dic. 2007; 61:3-27.

9. Cernadas A. La exclusión social en los grandes núcleos urbanos y el acceso a los servicios públicos por parte de las personas sin techo. In: Ponencia presentada en el X Congreso de AECPA. La política en la red. 7-9 sep. 2011; Murcia, España.

10. Max-Neef M. Desarrollo a escala humana. Conceptos, aplicaciones y algunas reflexiones. Barcelona: Icaria Editorial; 1998. 43 p.

11. Pomar A. Y Tendero G. Ja volem el pa sencer. Respostes a la pobresa alimentària en clau de Sobirania Alimentària. Barcelona: Aliança per a la Sobirania Alimentaria de Catalunya; 2015. [Acceso en 01 de abril de 2018]. Disponible en: http://xarxaconsum.net $/ \mathrm{mm} /$ file $/ \mathrm{Ja} \% 20$ volem $\% 20 \mathrm{el} \% 20 \mathrm{pa} \% 20$ sencer.pdf

12. Agurto F, Arteaga C y Pérez S. Subjetividades frente al riesgo: un estudio en familias urbanas vulnerables. Psicología, sociedad y equidad: aportes y desafíos. Universidad de Chile. 2012. p. 109-131. [acceso en 06 de abril de 2018] Disponible en: http://www.facso.uchile.cl/psicologia/publicaciones/ libros/libros_pdf/claudia_zuniga_psicologia_sociedad_equidad.pdf.

13. Bourdieu P, Director. La miseria del mundo. Madrid: Akal; 1999.

14. Castel R. La metamorfosis de la cuestión social. Una crónica del salariado. Barcelona: Paidós; 1997. 32 p.

15. Verthein U. Precarización social y alimentación: un análisis de los comedores sociales de la ciudad de Barcelona [Tesis]. [Barcelona]: Universidad de Barcelona, Departamento de Antropología Social, Facultad de Geografía e História; 2017.

Recibido: 09 de abril, 2018

Revisado: 22 de julio, 2018

Aceptado: 29 de julio, 2018 
